\title{
$\left[\mathrm{Co}(\mathrm{tp})_{2}(\mathrm{Me}-\mathrm{en})\right] \mathrm{ClO}_{4}$ 配合物不对称配 位氮原子的重氢化动力学研究
}

\author{
马桂林许宜铭 \\ (扬州师范学院化学系,扬州 225002)
}

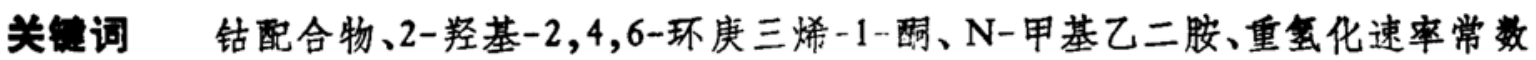

早在 1960 年, Palmer 等人曾认为: 水溶液中碱催化作用下八面体配合物中配位绻原子 的质子交换速率常数 $\left(k_{\mathrm{D}}\right.$, 重氢化速率常数)随配离子正电荷数的增多而增大 ${ }^{[\mathrm{d}}$. 近来, 我们 曾报道, 对于同属 $\mathrm{CoO}_{1} \mathrm{~N}_{2}$ 型的 $\left[\mathrm{Co}(\mathrm{OX})_{2}(\mathrm{Me}-\mathrm{en})\right]^{-}$和 $\left[\mathrm{Co}(\mathrm{acac})_{2}(\mathrm{Me}-\mathrm{en})\right]^{+}$配 离子 $\left(\mathrm{OX}-\mathrm{C}_{2} \mathrm{O}_{4}^{2-} ; \mathrm{acac}=2,4\right.$-戊二酮负离子; $\mathrm{Me}-\mathrm{en}=\mathrm{N}$-甲基乙二胺)来说, 虽然前者带有负 电荷, 但其不对称配位氮的重氢化速率常数 $k_{\mathrm{D}}$ 值却比带正电荷的后者的 $k_{\mathrm{D}}$ 值大 20 倍到. 为 进一步探讨影响重氢化速率的因素，我们合成了属 $\mathrm{CoO}_{4} \mathrm{~N}_{2}$ 型的 $\left[\mathrm{Co}(\mathrm{tp})_{2}(\mathrm{Me}-\mathrm{en})\right] \mathrm{ClO}_{4}$ 配 合物（tp - 2-羟基- 2,4,6-环庚三烯-1-酮负离子), 用高分辨核磁共振法测定了其不对称配 位货的 $k_{\mathrm{D}}$ 值, 并与 $\left[\mathrm{Co}(\mathrm{OX})_{2}(\mathrm{Me}-\mathrm{en})\right]^{-}$和 $\left[\mathrm{Co}(\mathrm{acac})_{2}(\mathrm{Me}-\mathrm{en})\right]^{+}$的 $k_{\mathrm{D}}$ 值进行了比较. 结 果表明,不对称配位氮的重氢化速率,比起配离子的电荷更易受其共存配体的影响以及不对称 配位氮上的氢原子所受空间位阻的影响.

\section{一、实验部 分}

1. 合成及元等分析 配体 2-羟基- $2,4,6$-环庚三烯-1-酮 (Htp)、原料配合物 $\left[\mathrm{Co}(\mathrm{tp})_{3}\right]$ 分别按文献[3]和 [4]类似方法合成。

$\Lambda(R) \Delta(S)$ 和 $\Lambda(S) \Delta(R)-\left[\mathrm{Co}(\mathrm{tp})_{2}(\mathrm{Me}-\mathrm{en})\right] \mathrm{ClO}_{4}$ 配合物按文献 [5] 类似方法合成及 异构体分离. $\Lambda(R) \Delta(S)$ 异构体元素分析值 (计算值, 计算式 $\left[\mathrm{Co}(\mathrm{tp})_{2}(\mathrm{Me}-\mathrm{en})\right] \mathrm{ClO}_{4} \cdot 0.5 \mathrm{H}_{2} \mathrm{O}$ $\left.-\mathrm{C}_{17} \mathrm{H}_{21} \mathrm{~N}_{2} \mathrm{ClCoO}_{8.5}\right)(\%): \mathrm{C}, 42.51(42.20) ; \mathrm{H}, 4.21(4.38) ; \mathrm{N}, 5.74(5.79) . \Lambda(S) \Delta(R)$ 异 构体元素分析值(计算值, 计算式 $\left.\left[\mathrm{Co}(\mathrm{tp})_{2}(\mathrm{Me}-\mathrm{en})\right] \mathrm{ClO}_{4} \cdot \mathrm{H}_{2} \mathrm{O}=\mathrm{C}_{17} \mathrm{H}_{22} \mathrm{~N}_{2} \mathrm{ClC}_{0} \mathrm{O}_{9}\right)(\%): \mathrm{C}$, 41.54(41.43); H, 4.44(4.51); N, 5.69(5.69).

2. 甲基质子化学位的测定用日立 R-90-HS 核磁共振仪测定 $\Lambda(R) \Delta(S)$ 和 $\Lambda(S) \Delta$ ( $R$ ) 异构体的甲基质子化学位移. 溶剂: $\mathrm{CD}_{3} \mathrm{OD}-\mathrm{DCl}$, 内标: TMS.

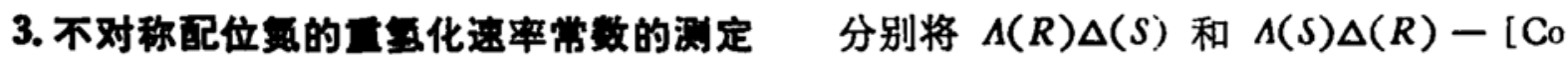
$(\mathrm{tp})_{2}(\mathrm{Me}-\mathrm{en}) \mathrm{C} \mathrm{ClO}$, 用 Dowex $1 \times 8\left(\mathrm{Cl}^{-}\right.$型) 阴离子交换树脂柱转化为溶解度较大的氯化 物后, 溶于磷酸盐 $-\mathrm{D}_{2} \mathrm{O}$ 缓冲液中 $\left(34^{\circ} \mathrm{C}, \mathrm{pH}-5.7-6.6\right)$, 使配合物浓度均为 $0.2 \mathrm{~mol} \cdot \mathrm{L}^{-1}$. 间隙取出部分反应液, 滴人少许 $\mathrm{DCl}-\mathrm{D}_{2} \mathrm{O}$ 溶液使其呈酸性 $(\mathrm{pH}-2 \sim 3)$, 然后用日立 $\mathrm{R}-$ 90-HS 核磁共振仪分别测定甲基质子核磁共振谱. 根据峰面积随时间的变化求得重氢化速 率常数 $k_{\mathrm{D}}$ 值.

\section{二、结果与 讨 论}

1. 具构体空间构型的推定 $\left[\mathrm{Co}(\mathrm{tp})_{2}(\mathrm{Me}-\mathrm{en})\right]^{+}$存在四种可能的旋光异构体 (图 1). 本文 1990 年 3 月 17 日收到. 1990 年 7 月 25 日收到修改稿. 


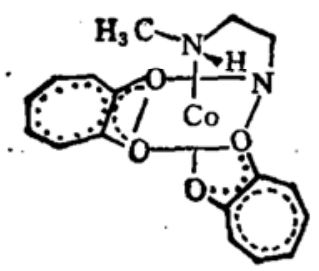

$\Delta(R)$

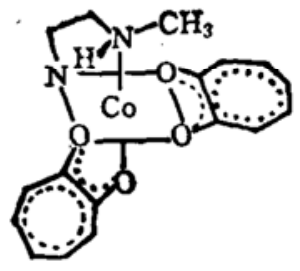

$\Lambda(S)$
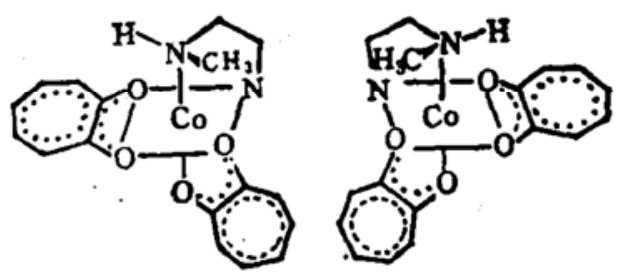

$\Delta(S)$

$\Lambda(R)$

图 $1\left[\mathrm{Co}(\mathrm{t} p)_{2}(\mathrm{Me}-\mathrm{en})\right]^{+}$的四个异构体

异构体的空间构型根据 ${ }^{1} \mathrm{HNMR}$ 法推定. 如图 1 所示, $\Lambda(S) \Delta(R)$ 异构体的甲基位于 一个 tp 螯合环上方, 由于螯合环的环电流效应, 该甲基受到较大的屏蔽作用, 甲基质子的化学 位移移向高场 $(\delta-1.84 \mathrm{ppm}, d, J-6 \mathrm{~Hz})$. 故将甲基质子的二重盽出现在高场的异构体推 定为 $\Lambda(S) \Delta(R)$ 构型. 同样将二重峰出现在低场 $(\delta-2.44 \mathrm{ppm}, d, J-6 \mathrm{~Hz})$ 的异构体推 定为 $\Lambda(R) \Delta(S)$ 构型.

2. 国化动力学方程 已有报道, $\left[\mathrm{Co}(\mathrm{acac})_{2}(\mathrm{Me}-\mathrm{en})\right]^{+[6]}$ 和 $\left[\mathrm{Co}(\mathrm{OX})_{2}(\mathrm{Me}-\mathrm{en})\right]^{-[2]}$ 在重水溶液中碱催化的重氢化(质子交换)反应相对于配合物浓度、OD- 离子浓度均为一级反 应, 总计为二级反应:

重氢化速率 $k-k_{\mathrm{D}}[$ 配合物 $]\left[O D^{-}\right]$.

本研究按类似方法处理. 重氢化反应可用下式表示(以 $\Lambda(R) \Delta(S)$ 异构体为例):

$$
\begin{aligned}
\Lambda(R) \Delta(S)-\left[\mathrm{Co}(\mathrm{tp})_{2}\left(\mathrm{CH}_{3} \mathrm{NHCH}_{2} \mathrm{CH}_{2} \mathrm{NH}_{2}\right)\right]^{+}+\mathrm{OD}^{-} \\
\quad \Lambda(R) \Delta(S)-\left[\mathrm{Co}(\mathrm{tp})_{2}\left(\mathrm{CH}_{3} \mathrm{NDCH}_{2} \mathrm{CH}_{2} \mathrm{NH}_{2}\right)\right]^{+}+\mathrm{OH}^{-} .
\end{aligned}
$$

该反应在一定 $\mathrm{pD}\left(\mathrm{pD}-\mathrm{pH}+0.4^{[\mathrm{J}}\right)$ 条件下可作为准一级反应处理:

$$
-\frac{d c_{A}}{d t}-k_{D\left(0 b_{3}\right) c_{A}}
$$

式中 $c_{A}$ 表示 $\Lambda(R) \Delta(S)-\left[\mathrm{Co}(\mathrm{tp})_{2}\left(\mathrm{CH}_{3} \mathrm{NHCH}_{2} \mathrm{CH}_{2} \mathrm{NH}_{2}\right)\right]^{+}$的浓度; $k_{D}($ obs $)$ 表示准一级反应 速率常数. 将(2)式积分得

$$
\ln \frac{c_{A}}{c_{A}}--k_{D\left(b_{0}\right) t},
$$

式中 $c_{\hat{A} 0}$ 和 $c_{\hat{A}}$ 分别表示反应物配合物的初始浓度和反应时间 $\boldsymbol{t}$ 的浓度.

设生成物 $\Lambda(R) \Delta(S)-\left[\mathrm{Co}(\mathrm{tp})_{2}\left(\mathrm{CH}_{3} \mathrm{NDCH}_{2} \mathrm{CH}_{2} \mathrm{NH}_{2}\right)\right]^{+}$的浓度为 $c_{\mathrm{B}}$. 据化学计量关系:

$$
c_{\mathrm{A}}+c_{\mathrm{B}}-c_{\mathrm{A} 0} \text { 及式(3)得 } \ln \left(1-\frac{c_{\mathrm{B}}}{c_{\mathrm{A}}+c_{\mathrm{B}}}\right)--k_{\mathrm{D}\left(\mathrm{ob}_{\mathrm{B}}\right) t .} \text {. }
$$

图 2 为 $34.0^{\circ} \mathrm{C} 、 \mathrm{pD}-6.85$ 条件下不同时刻测得的一组甲基质子核磁共振谱. 生成物浓 度 $c_{\mathrm{B}}$ 的变化相当位于二重峰中央单峰的变化. 故根据峰面积随时间变化率依式 (4) 可求得

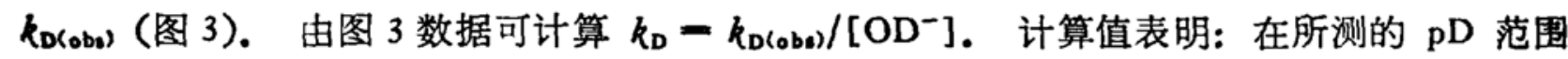
内, $k_{\mathrm{D}}$ 值基本上为一常数即二级反应速率常数. $k_{\mathrm{D}}$ 值列于表 1 .

3. 影响得化迹率常数的结构因意从表 1 可知, 对于 $\left[\mathrm{Co}(\mathrm{tp})_{2}(\mathrm{Me}-\mathrm{en})\right]^{+}$配离子, $\Lambda(S) \Delta(R)$ 异构体的 $k_{\mathrm{D}}$ 值大于 $\Lambda(R) \Delta(S)$ 异构体的 $k_{\mathrm{D}}$ 值. 根据这两种异构体的分子模型 可知, $k_{\mathrm{D}}$ 值的差异在于不对称配位氮原子上的氢原子所处空间位置的不同, $\mathrm{OD}^{-}$离子进攻该 氞原于时所受的空间位阻不同而造成. $\Lambda(R) \Delta(S)$ 异构体的不对称氮原子上的氢原子位于一 个 $\mathrm{tp}$ 整合环上方, 当 $\mathrm{OD}^{-}$离子向该氢原子接近时会受到鳌合环的较大空间位阻, 致使其 $k_{\mathrm{b}}$ 


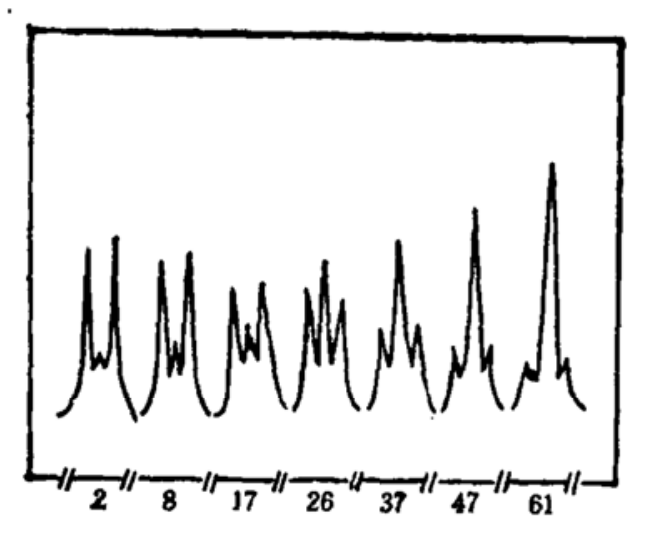

反应时间 $(\mathrm{min})$

固 $2 A(R) \Delta(S)-\left[\mathrm{Co}(\mathrm{p})_{2}(\mathrm{Me}-\mathrm{en})\right] \mathrm{Cl}$ 的 ${ }^{\prime} H N M R$ 谱随时间的变化

$\mathrm{PD}=6.85,34.0^{\circ} \mathrm{C}$

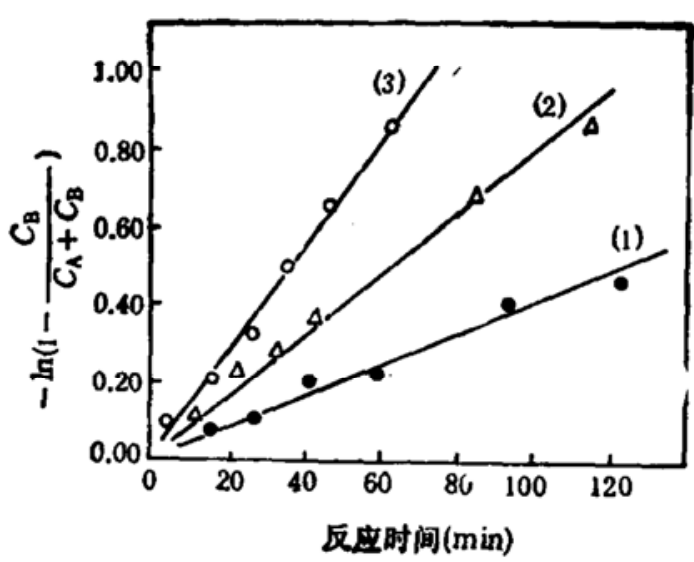

图 $3 A(R) \Delta(S)-\left[\mathrm{Co}(\mathrm{tp})_{2}(\mathrm{Me}-\mathrm{en})\right]^{+}$质子交 换准一级反应动力学曲线 $\left(34.0{ }^{\circ} \mathrm{C}\right)$

(1) $\mathrm{pD}=6.25$; (2) $\mathrm{pD}=6.67$; (3) $\mathrm{pD}=6.85$

值较小. 与此相对, $\Lambda(s) \Delta(R)$ 异构体的该氢原子位于 tp 鳌合环的侧上方, 有利于 $\mathrm{OD}^{-}$离 子接近而使 $k_{\mathrm{D}}$ 值较大.

表 $1\left[\mathrm{Co}(\mathrm{O}-\mathrm{O})_{2}(\mathrm{Me}-\mathrm{en})\right]^{+\mathrm{k}-}$ 的 $k_{\mathrm{D}}$ 值 $\left(34^{\circ} \mathrm{C}\right)$

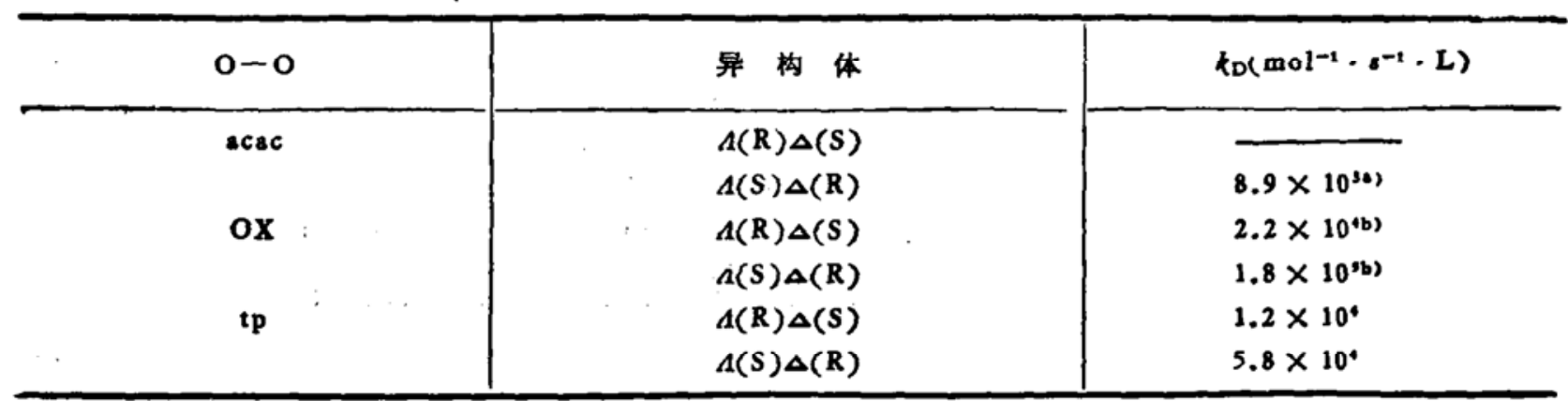

a) 和 b) 数据分别引自文嗝[6]和[2].

具有相同 $\mathrm{CoO}_{4} \mathrm{~N}_{2}$ 型的 $\left[\mathrm{Co}(\mathrm{tp})_{2}(\mathrm{Me}-\mathrm{en})\right]^{+}$、 $\left[\mathrm{Co}(\mathrm{acac})_{2}(\mathrm{Me}-\mathrm{en})\right]^{+}$和 $\left[\mathrm{Co}(\mathrm{OX})_{2}(\mathrm{Me}-\right.$ en) $]^{-}$的 $k_{\mathrm{D}}$ 值 (表 1) 亦有差异. 已有报道, 配合物的 $k_{\mathrm{D}}$ 值随配离子正电荷的增多而增大 ${ }^{[\mathrm{l}}$, 但本研究的情况恰恰相反. $\left[\mathrm{Co}(\mathrm{tp})_{2}(\mathrm{Me}-\mathrm{en})\right]^{+}$的 $k_{\mathrm{D}}$ 值比 $\left[\mathrm{Co}(\mathrm{OX})_{2}(\mathrm{Me}-\mathrm{en})\right]^{-}$的 $k_{\mathrm{D}}$ 值为 小, $\left[\mathrm{Co}(\mathrm{acac})_{2}(\mathrm{Me}-\mathrm{en})\right]^{+}$与 $\left[\mathrm{Co}(\mathrm{OX})_{2}(\mathrm{Me}-\mathrm{en})\right]^{-}$的 $k_{\mathrm{D}}$ 值也出现类似变化情况. $k_{\mathrm{D}}$ 值相 对大小次序为: $\left[\mathrm{Co}(\mathrm{OX})_{2}(\mathrm{Me}-\mathrm{en})\right]^{-}>\left[\mathrm{Co}(\mathrm{tp})_{2}(\mathrm{Me}-\mathrm{en})\right]^{+}>\left[\mathrm{Co}(\mathrm{acac})_{2}(\mathrm{Me}-\mathrm{en})\right]^{+}$. 这表 明, 比起配离子的电荷, $k_{\mathrm{D}}$ 值更易受共存配体的影响.

tp 以及 acac 为配体的钴配合物具有较小的 $k_{\mathrm{D}}$ 值的原因正在进一步研究之中. 不过值得 注意的是, 由 $t p$ 以及 $\mathrm{acac}$ 形成的鳌合环均具有离域 $\pi$ 电子体系.

\section{梦考文献}

[1] Palmer, J.W. and Basolo, F., J. Phys. Chem., 64(1960), 778-780; Basolo, F. et al., J. Am. Chem. Soc., 82(1960), 1073-1076.

[2] Ma, G., Hibino, T. et al., Bull. Chem. Soc. Jpn., 62(1989), 1053-1056.

[3] Minns, R.A., Orgn. Synih., 57(1977),117.

[4] Shibata. M., Nippon Kagakw Zasshi, 87(1966),771.

[5] Ouyang, Y., Kojima, M. et al., Bull. Chem. Soc. Jpn., 57(1984),3574-3579.

[6] Kojima, M., Hibino, T. et al., Inorg. Chim. Acta, 117 (1986),1-4.

[7] Glson, F. K., Long, F. A., J. Phys. Chem., 64(1960), 188-190. 